\title{
Efeitos do Raloxifeno sobre a Concentração Plasmática de Homocisteína e 0 Lipidograma em Mulheres na Pós-Menopausa
}

\author{
Effects of Raloxifene on Plasma Homocysteine Concentration and Lipid Profile in \\ Postmenopausal Women
}

Márcia Neves de Carvalho ${ }^{1}$, George Dantas de Azevedo ${ }^{1}$, José Ernesto dos Santos ${ }^{2}$, Rui Alberto Ferriani ${ }^{1}$, Rosana Maria dos Reis ${ }^{1}$, Marcos Felipe Silva de Sá ${ }^{1}$

\begin{abstract}
RESUM0
Objetivos: avaliar os efeitos do raloxifeno sobre a concentração plasmática de homocisteína e o perfil lipídico em mulheres na pós-menopausa.

Métodos: foram estudadas 24 mulheres saudáveis na pós-menopausa, com idade entre 50 e 70 anos e diagnóstico de osteopenia e/ou osteoporose, submetidas à terapia com raloxifeno, $60 \mathrm{mg} /$ dia, durante seis meses. Foram dosados a homocisteína plasmática antes do início e após três e seis meses de tratamento, além do colesterol total, HDL-colesterol, LDL-colesterol e triglicérides. A concentração de homocisteína foi determinada por imunoensaio de fluorescência polarizada e o lipidograma pelo método colorimétrico e enzimático. Para a análise estatistica foram utilizados os testes ANOVA, de Newman-Keuls e de correlação de Pearson. Resultados: observamos redução significativa do colesterol total de 15,3\% (227,6士56,3 vs $200,6 \pm 29,8$ vs $192,8 \pm 32,1 \mathrm{mg} / \mathrm{dL} ; p<0,001)$ e do LDL-colesterol de $21,4 \%(151,4 \pm 46,3 \mathrm{vs}$ $122,7 \pm 29,4$ vs $119,0 \pm 28,6 \mathrm{mg} / \mathrm{dL} ; p<0,001)$ e aumento significativo do HDL-colesterol de $9,5 \%(44,7 \pm 10,8$ vs $52,2 \pm 12,6$ vs $49,0 \pm 10,8 \mathrm{mg} / \mathrm{dL} ; p<0,05)$. Não houve redução significativa dos triglicérides $(134,9 \pm 50,3$ vs $127,5 \pm 50,0$ vs $121,0 \pm 36,0 \mathrm{mg} / \mathrm{dL} ; p>0,05)$. Embora não significativa, foi observada redução da homocisteína de $4.5 \%(11,7 \pm 3,0$ vs $11,0 \pm 2,9$ vs $11,2 \pm 2,1 \mu \mathrm{M} / L ; p>0,05)$ entre os periodos pré e pós-tratamento, com correlação negativa significativa entre os níveis basais e os percentuais de redução após o tratamento $(r=-0,71$; $p<0,0001$ ).

Conclusões: a terapia com raloxifeno, $60 \mathrm{mg} / \mathrm{dia}$, em mulheres na pós-menopausa durante seis meses levou a reduções do colesterol total e do LDL-colesterol e aumento do HDLcolesterol. Houve tendência à redução dos níveis plasmáticos de homocisteína, sendo o efeito mais favorável nas pacientes com níveis basais mais elevados.
\end{abstract}

PALAVRAS-CHAVE: Menopausa. Doença cardiovascular. Raloxifeno. Homocisteína. Perfil lipídico.

\footnotetext{
${ }^{1}$ Setor de Reprodução Humana do Departamento de Ginecologia e Obstetrícia, ${ }^{2}$ Divisão de Nutrologia do Departamento de Clínica Médica da Faculdade de Medicina de Ribeirão Preto da Universidade de São Paulo Correspondência:

Marcos Felipe Silva de Sá

Endereço: Departamento de Ginecologia e Obstetrícia

Av. Bandeirantes 3900

14049-900 - Ribeirão Preto - SP

Telefone: (16) 602-2817

Fax: (16) 633-9633

e-mail: mfsdsa@fmrp.usp.br
}

\section{Introdução}

A doença cardiovascular (DCV), particularmente a doença arterial coronariana (DAC), é a principal causa de mortalidade entre as mulheres. O fato de mulheres, após a menopausa, apresentarem aumento importante na incidência de DAC sugere que o hipoestrogenismo esteja envolvido na etiologia dessa doença. Essa hipótese foi 
reforçada durante longos anos por relatos de redução do risco de DAC em mulheres menopausadas submetidas à terapia hormonal (TH).

Entretanto, resultados dos últimos estudos sobre os efeitos da TH sobre o risco cardiovascular contradizem os dados anteriormente citados, gerando controvérsias ${ }^{1,2}$. Diante desses novos dados da literatura, a busca por alternativas terapêuticas à TH tornou-se necessária. Uma das opções disponíveis são os moduladores seletivos do receptor estrogênico (SERMs).

O raloxifeno é um SERM de segunda geração, que tem papel bem estabelecido na prevenção e tratamento da osteoporose que ocorre após a menopausa e vem surgindo como droga promissora na prevenção do câncer de mama ${ }^{3}$. Entretanto, os efeitos do raloxifeno sobre a evolução da DCV ainda não estão bem estabelecidos.

A homocisteína é aminoácido que contém enxofre e o aumento da sua concentração plasmática é atualmente reconhecido como um fator de risco independente para DCV, incluindo aterosclerose prematura e aterotrombose coronariana, cerebral e periférica ${ }^{4}$. A hiperhomocisteinemia causa lesão e disfunção endoteliais por toxicidade direta e aumento dos níveis de moléculas de adesão circulantes e de marcadores de coagulação, potencialmente mediante efeito pró-oxidante no endotélio vascular ${ }^{5,6}$.

Considerando o raloxifeno como terapêutica alternativa à TH, e em face da escassez de dados acerca dos seus efeitos sobre o sistema cardiovascular, o objetivo desse estudo foi avaliar os efeitos da terapia com raloxifeno sobre a concentração plasmática de homocisteína e o lipidograma em mulheres na pós-menopausa.

\section{Pacientes e Métodos}

O estudo foi realizado no Departamento de Ginecologia e Obstetrícia da Faculdade de Medicina de Ribeirão Preto da Universidade de São Paulo (FMRP - USP) e teve a aprovação do Comitê de Ética em Pesquisa do Hospital das Clínicas da FMRP - USP. Todas as pacientes assinaram termo de consentimento pós-informação.

Vinte e quatro mulheres saudáveis na pósmenopausa foram selecionadas no período entre agosto de 2001 e julho de 2002. Os critérios de inclusão foram: idade entre 50 e 70 anos; último ciclo menstrual espontâneo há mais de 12 meses; concentração sérica de hormônio folículo-estimulante $(\mathrm{FSH})$ maior que $40 \mathrm{UI} / \mathrm{mL}$; diagnóstico de osteopenia e/ou osteoporose e a não utilização de $\mathrm{TH}$ nos 90 dias imediatamente anteriores à inclusão no estudo.

Os critérios de exclusão foram história ou presença de hipertensão arterial, doença cardiovascular, distúrbio tromboembólico, doença endócrina ou metabólica, neoplasia estrogêniodependente, síndrome de má absorção, alcoolismo ou drogadição, obesidade [índice de massa corpórea (IMC) maior ou igual a $\left.30\left(\mathrm{~kg} / \mathrm{m}^{2}\right)\right] \mathrm{e} / \mathrm{ou}$ alterações nos seguintes exames laboratoriais: hemograma, urina rotina, glicemia de jejum, alanina e aspartato aminotransferases, bilirrubinas, uréia e creatinina.

As pacientes selecionadas apresentaram as seguintes características: idade de 56,0 $\pm 4,8$ anos, tempo de menopausa de $8,2 \pm 5,1$ anos, IMC de $25,2 \pm 3,6$, PA sistólica de $125,6 \pm 17,2 \mathrm{mmHg}$, PA diastólica de $80,5 \pm 8,0 \mathrm{mmHg}$ e níveis de FSH de $79,1 \pm 35,5 \mathrm{mUI} / \mathrm{mL}$. A caracterização completa da casuística é apresentada na Tabela 1.

Tabela 1 - Características clínicas de mulheres na pós-menopausa antes do tratamento com raloxifeno $(60 \mathrm{mg} / \mathrm{dia})$.

\begin{tabular}{lc}
\hline Característica & \\
\hline Idade (anos) & $56,0 \pm 4,8$ \\
Tempo de menopausa (anos) & $8,2 \pm 5,1$ \\
Peso $(\mathrm{kg})$ & $62,8 \pm 11,8$ \\
Estatura $(\mathrm{cm})$ & $157,7 \pm 7,2$ \\
Índice de massa corporal $\left(\mathrm{kg} / \mathrm{m}^{2}\right)$ & $25,2 \pm 3,6$ \\
Pressão arterial sistólica $(\mathrm{mmHg})$ & $125,6 \pm 17,2$ \\
Pressão arterial diastólica $(\mathrm{mmHg})$ & $80,5 \pm 8,0$ \\
Freqüência cardíaca (bpm) & $71.6 \pm 6.7$ \\
Relação cintura/quadril & $0,81 \pm 0,04$ \\
FSH (mUl/mL) & $79.1 \pm 35.5$ \\
\hline
\end{tabular}

Dados expressos como média \pm desvio-padrão.

Foi fornecido às pacientes o cloridrato de raloxifeno em quantidade suficiente para seis meses de uso. A medicação foi prescrita na dosagem de $60 \mathrm{mg}$ (um comprimido) ao dia por via oral e as pacientes foram orientadas quanto às medidas higieno-dietéticas, de acordo com a rotina do serviço.

As coletas sanguíneas foram realizadas antes do início do tratamento e após três e seis meses do uso da medicação. As pacientes compareceram ao hospital entre as 8:00 e 10:00 horas da manhã, após período de jejum de dez horas. As amostras sanguíneas foram obtidas por punção venosa antecubital e a seguir centrifugadas a 1000 $g$ durante dez minutos. As amostras de plasma 
obtidas foram, então, congeladas à temperatura de $20^{\circ} \mathrm{C}$ negativos até a realização dos ensaios.

Os níveis de homocisteína foram avaliados utilizando-se o kit IMx Metabólicos - Homocisteína, fabricado por Abbott Laboratórios do Brasil. As dosagens foram realizadas no Laboratório de Nutrição do Hospital das Clínicas da FMRP - USP. O método utilizado foi o imunoensaio de fluorescência polarizada para a determinação quantitativa de L-homocisteína total no plasma. Para evitar o erro interensaio, as três amostras de cada paciente (basal, 90 dias e 180 dias) foram analisadas num mesmo ensaio.

Para a determinação das concentrações de colesterol total, HDL-colesterol, LDL-colesterol e triglicérides, as amostras sanguíneas foram encaminhadas ao laboratório acima citado, imediatamente após a coleta. As dosagens foram realizadas utilizando-se kits de fabricação Roche Produtos Químicos e Farmacêuticos S/A. O método utilizado foi colorimétrico e enzimático.

A análise estatística dos níveis de homocisteína foi realizada utilizando-se a análise de variância para medidas repetidas (ANOVA) para a comparação entre os valores obtidos antes do tratamento e aqueles referentes às fases posteriores da pesquisa (90 e 180 dias). O nível de significância adotado foi de 5\%. O teste de correlação de Pearson foi utilizado posteriormente para avaliar a correlação entre os niveis basais de homocisteína e os percentuais de variação entre os níveis basais e os niveis após 180 dias de tratamento.

Para a análise estatística do lipidograma, foi utilizado o teste ANOVA para todos os parâmetros estudados, visando a comparação entre os valores pré e pós-tratamento. O teste de Newman-Keuls foi utilizado posteriormente para avaliação do colesterol total, LDL-colesterol e HDL-colesterol. Foram utilizados para a análise estatística os softwares Prism 3 e GraphPad StatMate.

\section{Resultados}

A média \pm DP dos valores de homocisteína plasmática antes do início do tratamento foi de $11,7 \pm 3,0 \mu \mathrm{M} / \mathrm{L}$, com uma variação de 6,0 a 19,9. Após 90 dias de tratamento, essa média foi de $11,0 \pm 2,9 \mu \mathrm{M} / \mathrm{L}$, com variação de 5,7 a 17,3. Após 180 dias de tratamento, esse valor foi de $11,2 \pm 2,1$ $\mu \mathrm{M} / \mathrm{L}$, com uma variação de 6,8 a 15,1 .

Observamos tendência à redução dos níveis de homocisteína após 90 e 180 dias de tratamento com raloxifeno, em média de 0,73 e 0,54 $\mu \mathrm{M} / \mathrm{L}$, respectivamente. Estas diferenças não são significativas, quando comparados os níveis de homocisteína nas três fases do estudo $(11,7 \pm 3,0$ vs $11,0 \pm 2,9$ vs $11,2 \pm 2,1 \mu \mathrm{M} / \mathrm{L} ; \mathrm{p}>0,05)$. Em termos percentuais, a redução média foi de $4,5 \%$ em relação aos valores iniciais. A análise mostrou correlação negativa entre os níveis basais de homocisteína e os percentuais de redução após 180 dias de administração do raloxifeno $(\mathrm{r}=-0,71$; $\mathrm{p}<0,0001$ ), ou seja, quanto maior o nível basal, maior a redução observada (Figura 1).

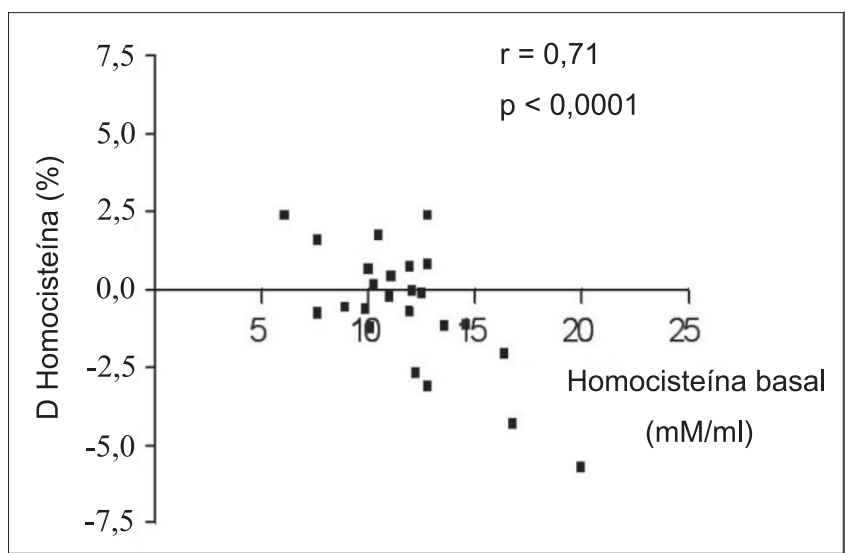

Figura 1 - Correlação entre os níveis basais de homocisteína plasmática e os seus percentuais de variação após 180 dias de uso do raloxifeno (60 mg/dia) em mulheres na pós-menopausa.

Assim, quando analisamos somente as pacientes com valores basais superiores a $12,8 \mu \mathrm{M}$ / $\mathrm{L}$, referido como limite da normalidade para mulheres com mais de 50 anos $^{7}$, obtivemos valor basal médio de $16,2 \mu \mathrm{M} / \mathrm{L}$ e verificamos que a redução média após 180 dias de tratamento foi de $2.8 \mu \mathrm{M}$ / L, cerca de cinco vezes, em valores absolutos, aquela observada no grupo total. Em termos percentuais, a redução naquele subgrupo foi de $17,5 \%$, cerca de quatro vezes maior que a do grupo total.

$\mathrm{Na}$ avaliação do lipidograma, verificou-se redução significativa de $15,3 \%$ no colesterol total $(227,6 \pm 56,3$ vs $200,6 \pm 29,8$ vs $192,8 \pm 32,1 \mathrm{mg} / \mathrm{dL}$; $\mathrm{p}<0,001)$ e de $21,4 \%$ no LDL-colesterol $(151,4 \pm 46,3$ vs $122,7 \pm 29,4$ vs $119,0 \pm 28,6 \mathrm{mg} / \mathrm{dL} ; \mathrm{p}<0,001)$. Houve, em contrapartida, aumento significativo de 9.5\% no HDL-colesterol $(44,7 \pm 10,8$ vs $52,2 \pm 12,6$ vs $49,0 \pm 10,8 \mathrm{mg} / \mathrm{dL} ; \mathrm{p}<0,05)$. Para os triglicérides, a redução não foi significativa $(134,9 \pm 50,3$ vs $127,5 \pm 50,0$ vs $121,0 \pm 36,0 \mathrm{mg} / \mathrm{dL} ; \mathrm{p}>0,05)$. Os níveis de homocisteína, colesterol total, HDLcolesterol, LDL-colesterol e triglicérides antes e após 90 e 180 dias de tratamento com raloxifeno são apresentados na Tabela 2. 
Tabela 2 - Níveis plasmáticos de homocisteína e lipidograma de mulheres na pósmenopausa antes e após 90 e 180 dias de tratamento com raloxifeno (60 mg/dia).

\begin{tabular}{lccc}
\hline Parâmetro medido & Basal & $\begin{array}{c}90 \text { dias de } \\
\text { tratamento }\end{array}$ & $\begin{array}{c}180 \text { dias de } \\
\text { tratamento }\end{array}$ \\
\hline Homocisteína $(\mu \mathrm{M} / \mathrm{l})$ & $11,7 \pm 3,0$ & $11,0 \pm 2,9$ & $11,2 \pm 2,1$ \\
& $(6,0-19,9)$ & $(5,7-17,3)$ & $(6,8-15,1)$ \\
Colesterol total $(\mathrm{mg} / \mathrm{dL})$ & $227,6 \pm 56,3$ & $200,6 \pm 29,8^{\star *}$ & $192,8 \pm 32,1^{* * *}$ \\
& $(137,0-356,0)$ & $(156,0-277,0)$ & $(143,0-262,0)$ \\
HDL-colesterol $(\mathrm{mg} / \mathrm{dL})$ & $44,7 \pm 10,8$ & $52,2 \pm 12,6^{\star * *}$ & $49,0 \pm 10,8^{*}$ \\
& $(21,6-65,0)$ & $(36,0-90,4)$ & $(27,7-72,0)$ \\
LDL-colesterol $(\mathrm{mg} / \mathrm{dL})$ & $151,4 \pm 46,3$ & $122,7 \pm 29,4^{\star * *}$ & $119,0 \pm 28,6^{\star * *}$ \\
& $(76,0-257,0)$ & $(83,0-202,0)$ & $(70,0-181,0)$ \\
Triglicérides $(\mathrm{mg} / \mathrm{dL})$ & $134,9 \pm 50,3$ & $127,5 \pm 50,0$ & $121,0 \pm 36,0$ \\
& $(80,0-290,0)$ & $(72,0-299,0)$ & $(58,0-211,0)$ \\
\hline
\end{tabular}

Dados expressos como média \pm desvio-padrão.

${ }^{*} p<0.05$ em relação ao basal.

${ }^{* *} p<0.01$ em relação ao basal.

${ }^{* * *} p<0.001$ em relação ao basal.

\section{Discussão}

O raloxifeno é SERM de segunda geração, cujos efeitos sobre o sistema cardiovascular ainda estão por ser definidos. Alguns estudos clínicos sugerem que o raloxifeno tem influência positiva sobre os marcadores sanguíneos lipídicos da DCV, levando à redução do colesterol total e do LDL-colesterol, sem apresentar efeito, entretanto, sobre as concentrações dos triglicérides ${ }^{3,8,9}$. Quanto ao HDL-colesterol, os dados são contraditórios, relatando-se tanto aumento ${ }^{10}$ como ausência de efeito ${ }^{3,9,11,12}$. O resultado de uma metanálise ${ }^{13}$ mostrou que o uso do raloxifeno está associado a um aumento do HDL-colesterol de 1,5 a 5,7\%, embora em nossos resultados os aumentos percentuais tenham sido maiores que estes $(9,5$ a $16,6 \%)$.

Nas mulheres, o principal indicador de DAC é nível baixo de HDL-colesterol ${ }^{14}$, e sua elevação (mais de $55 \mathrm{mg} / \mathrm{dL}$ ) pode neutralizar os efeitos adversos do colesterol total e do LDL-colesterol em altas concentrações ${ }^{15}$. Além disso, o nível de HDLcolesterol está inversamente relacionado à mortalidade por DCV, particularmente por DAC, demonstrando assim a importância desse fator na epidemiologia dessas doenças ${ }^{14}$.

Estudos experimentais demonstram que a homocisteína aumenta a captação e a oxidação do LDL-colesterol na parede arterial e estimula a proliferação das células musculares lisas do vaso, processos de importância fundamental na gênese da aterosclerose ${ }^{16}$. Além disso, a homocisteína tem ação trombogênica, inibindo a expressão da trombomodulina e a ativação da proteína $\mathrm{C}^{17} \mathrm{e}$ aumentando a atividade do fator XII e do fator $\mathrm{V}^{5}$.

Os fatores que interferem nos níveis plasmáticos de homocisteína são de origem genética e nutricional ${ }^{18,19}$. Existem evidências de que os estrogênios também interferem nos níveis desse aminoácido. A baixa concentração de homocisteína em mulheres na pré-menopausa e o seu subseqüente aumento após esse período sugerem associação entre o seu metabolismo e os niveis estrogênicos ${ }^{20}$.

Uma diminuição da vasodilatação endotéliodependente foi observada em pessoas idosas com hiper-homocisteinemia, sugerindo redução da biodisponibilidade do óxido nítrico ${ }^{21}$. Um estudo realizado recentemente evidenciou que o uso do raloxifeno em mulheres menopausadas saudáveis está associado a efeito protetor da função endotelial, levando a modulação positiva sobre a vasodilatação endotélio-dependente ${ }^{22}$.

Embora alguns autores relatem redução dos níveis de homocisteína com o uso do raloxifeno, de modo geral as doses utilizadas são superiores (120 a $150 \mathrm{mg} /$ dia) àquelas habitualmente empregadas na clínica, o que limita a indicação do uso para obtenção deste benefício do medicamento $^{23,24}$. Nas doses habituais de raloxifeno $(60 \mathrm{mg} /$ dia), os trabalhos falham em apontar redução significativa dos níveis plasmáticos de homocisteína ${ }^{23,25}$.

Além do fator dose-dependência, outras variáveis têm sido motivo de controvérsias. Pacientes mais idosas (60-70 anos) parecem responder melhor à terapia ${ }^{26}$ do que aquelas menopausadas há pouco tempo ${ }^{25}$. O tempo de exposição à droga também pode influenciar, pois a terapia mais prolongada pode dar melhores resultados do que períodos curtos de tratamento ${ }^{26}$. Os dados mais recentes da literatura ${ }^{27}$ relatam que o uso do raloxifeno, na dose de $60 \mathrm{mg} /$ dia durante 18 meses, tem efeito significativo sobre as concentrações de homocisteína e que a magnitude da redução está diretamente relacionada à duração da terapia. Nosso estudo mostrou que, após seis meses de tratamento com raloxifeno na dose de $60 \mathrm{mg} / \mathrm{dia}$, houve redução, embora não significativa, das concentrações plasmáticas de homocisteína em pacientes pós-menopausadas saudáveis. A ausência de significância, encontrada tanto neste quanto em alguns outros trabalhos apresentados na literatura $^{23,25}$, poderia ser justificada pelos valores basais encontrados, pois quando avaliamos apenas o subgrupo de pacientes com niveis basais elevados, encontramos reduções mais significativas da homocisteína tanto em valores absolutos como percentuais. 
Finalmente, analisando os nossos resultados juntamente com os da literatura ${ }^{26,27}$, poderíamos concluir que o raloxifeno tem efeito benéfico sobre o metabolismo lipídico, e levantar a hipótese de que a redução dos níveis de homocisteína pelo uso do raloxifeno depende da dose utilizada dessa medicação e dos valores basais de homocisteína. Uma vez que a dosagem de raloxifeno utilizada na prática clínica é de $60 \mathrm{mg} /$ dia, poderíamos sugerir que essa medicação teria melhores resultados quanto à redução dos níveis de homocisteína se indicada para pacientes com niveis basais mais elevados.

\section{ABSTRACT}

Purpose: to evaluate the effects of raloxifene on plasma homocysteine concentration and lipid profile in postmenopausal women.

Methods: twenty-four healthy postmenopausal women, aged 50 to 70 years, with osteopenia and/or osteoporosis, were submitted to raloxifene therapy, $60 \mathrm{mg} /$ day, for six months. Plasma homocysteine concentration was determined before and after three and six months of therapy, as well as total cholesterol, HDL-cholesterol LDL-cholesterol and triglyceride levels. Plasma homocysteine was measured by a polarized immunofluorescence assay and serum lipids by the enzymatic and colorimetric method. Data were analyzed statistically by ANOVA, Newman-Keuls test and Pearson's correlation test.

Results: a significant decrease in total cholesterol of $15.3 \%$ $(227.6 \pm 56.3$ vs $200.6 \pm 29.8$ vs $192.8 \pm 32.1 \mathrm{mg} / \mathrm{dL} ; p<0.001)$ and $L D L$-cholesterol of $21.4 \%(151.4 \pm 46.3$ vs $122.7 \pm 29.4$ vs $119.0 \pm 28.6 \mathrm{mg} / \mathrm{dL} ; p<0.001$ ), and a significant increase in HDL-cholesterol of $9.5 \%(44.7 \pm 10.8$ vs $52.2 \pm 12.6$ vs $49.0 \pm 10.8 \mathrm{mg} / \mathrm{dL} ; p<0.05)$ were observed. There was no reduction in triglyceride levels $(134.9 \pm 50.3$ vs $127.5 \pm 50.0$ vs $121.0 \pm 36.0 \mathrm{mg} / \mathrm{dL} ; p>0.05$ ). Although not significant, a decrease in homocysteine by $4.5 \%(11.7 \pm 3.0$ vs $11.0 \pm 2.9$ vs $11.2 \pm 2.1 \mu M / L ; p>0.05)$ was observed between the pre-and posttreatment periods, with a significant negative correlation between basal levels and posttreatment percentual reduction $(r=-0.71 ; p<0.0001)$.

Conclusions: raloxifene treatment, $60 \mathrm{mg} /$ day, for six months caused a significant decrease in total and LDL-cholesterol and an increase in HDL-cholesterol in postmenopausal women. Plasma homocysteine concentration tended to decrease, this effect being more favorable in patients with elevated baseline levels.

KEYWORDS: Menopause. Cardiovascular disease. Raloxifene. Homocysteine. Lipid profile.

\section{Referências}

1. Grady D, Herrington D, Bittner V, et al. Cardiovascular disease outcomes during 6.8 years of hormone therapy: Heart and Estrogen/progestin Replacement Study follow-up (HERS II). JAMA 2002; 288:49-57.

2. Rossouw JE, Anderson GL, Prentice RL, et al. Risks and benefits of estrogen plus progestin in healthy postmenopausal women: principal results From the Women's Health Initiative randomized controlled trial. JAMA 2002; 288:321-33.

3. Cummings SR, Eckert S, Krueger KA, et al. The effect of raloxifene on risk of breast cancer in postmenopausal women: results from the MORE randomized trial. Multiple Outcomes of Raloxifene Evaluation. JAMA 1999; 281:2189-97.

4. Welch GN, Loscalzo J. Homocysteine and atherothrombosis. N Engl J Med 1998; 338:1042-50.

5. Chambers JC, McGregor A, Jean-Marie J, Obeid OA, Kooner JS. Demonstration of rapid onset vascular endothelial dysfunction after hyperhomocysteinemia: an effect reversible with vitamin $\mathrm{C}$ therapy. Circulation 1999; 99:1156-60.

6. Nappo F, De Rosa N, Marfella R, et al. Impairment of endothelial functions by acute hyperhomocysteinemia and reversal by antioxidant vitamins. JAMA 1999; 281:2113-8.

7. Andersson A, Brattstrom L, Israelsson B, Isaksson A, Hamfelt A, Hultberg B. Plasma homocysteine before and after methionine loading with regard to age, gender, and menopausal status. Eur J Clin Invest 1992; 22:79-87.

8. Lufkin EG, Whitaker MD, Nickelsen T, et al. Treatment of established postmenopausal osteoporosis with raloxifene: a randomized trial. J Bone Miner Res 1998; 13:1747-54.

9. Walsh BW, Kuller LH, Wild RA, et al. Effects of raloxifene on serum lipids and coagulation factors in healthy postmenopausal women. JAMA 1998; 279:1445-51.

10.Nickelsen T, Creatsas G, Rechberger T, et al. Differential effects of raloxifene and continuous combined hormone replacement therapy on biochemical markers of cardiovascular risk: results from the Euralox 1 study. Climacteric 2001; 4:320-31.

11.Barrett-Connor E, Ensrud KE, Harper K, et al. Post hoc analysis data from the Multiple Outcomes of Raloxifene Evaluation (MORE) trial on the effects of three years of raloxifene treatment on glycemic control and cardiovascular disease risk factors in women with and without type 2 diabetes. Clin Ther 2003; 25:919-30.

12.Jolly EE, Bjarnason NH, Neven P, et al. Prevention of osteoporosis and uterine effects in postmenopausal women taking raloxifene for 5 years. Menopause 2003; 10:337-44. 
13.Umland EM, Rinaldi C, Parks SM, Boyce EG. The impact of estrogen replacement therapy and raloxifene on osteoporosis, cardiovascular disease, and gynecologic cancers. Ann Pharmacother 1999; 33:1315-28.

14.Jacobs DR Jr, Mebane IL, Bangdiwala SI, Criqui MH, Tyroler HA. High density lipoprotein cholesterol as a predictor of cardiovascular disease mortality in men and women: the follow-up study of the Lipid Research Clinics Prevalence Study. Am J Epidemiol 1990; 131:32-47.

15.Blankenhorn DH, Nessim SA, Johnson RL, Sanmarco ME, Azen SP, Cashin-Hemphill L. Beneficial aspects of combined colestipol-niacin therapy on coronary atherosclerosis and coronary venous bypass graft. JAMA 1987; 257:3233-40.

16.Rees MM, Rodgers GM. Homocysteinemia: association of a metabolic disorder with vascular disease and thrombosis. Thromb Res 1993; 71:337-59.

17.Harpel PC, Zhang X, Borth W. Homocysteine and hemostasis: pathogenic mechanisms predisposing to thrombosis. J Nutr 1996; 126 Suppl:1285S-9S.

18. Mudd SH, Levy HL, Skovby F. Disorders of transsulfuration. In: Scriver CR, Beaudet AL, Sly WS, Valle D, editors. The Metabolic Basis of Inherited Disease. $6^{\text {th }}$ ed. New York: McGraw-Hill; 1989. p. 693-734.

19.Jacobsen DW. Homocysteine and vitamins in cardiovascular disease. Clin Chem 1998; 44:1833-43.

20.Wouters MG, Moorrees MT, van der Mooren MJ, et al. Plasma homocysteine and menopausal status. Eur J Clin Invest 1995; 25:801-5.

21.Tawakol A, Omland T, Gerhard M, Wu JT, Creager MA. Hyperhomocyst(e)inemia is associated with impaired endothelium-dependent vasodilation in humans. Circulation 1997; 95:1119-21.

22. Colacurci N, Manzella D, Fornaro F, Carbonella M, Paolisso G. Endothelial function and menopause: effects of raloxifene administration. J Clin Endocrinol Metab 2003; 88:2135-40.

23.Mijatovic V, Netelenbos C, van der Mooren MJ, de Valk-de Roo GW, Jakobs C, Kenemans P. Randomized, double-blind, placebo-controlled study of the effects of raloxifene and conjugated equine estrogen on plasma homocysteine levels in healthy postmenopausal women. Fertil Steril 1998; 70:1085-9.

24.Walsh BW, Paul S, Wild RA, et al. The effects of hormone replacement therapy and raloxifene on $\mathrm{C}$ reactive protein and homocysteine in healthy postmenopausal women: a randomized, controlled trial. $\mathrm{J}$ Clin Endocrinol Metab 2000; 85:214-8.

25.Smolders RG, Vogelvang TE, Mijatovic V, et al. A 2year, randomized, comparative, placebo-controlled study on the effects of raloxifene on lipoprotein(a) and homocysteine. Maturitas 2002; 41:105-14.

26.De Leo V, la Marca A, Morgante G, Lanzetta D, Setacci C, Petraglia F. Randomized control study of the effects of raloxifene on serum lipids and homocysteine in older women. Am J Obstet Gynecol 2001; 184:350-3.

27.Christodoulakos G, Lambrinoudaki I, Panoulis C, Rizos D, Coutoukos J, Creatsas G. Effect of raloxifene, estrogen, and hormone replacement therapy on serum homocysteine levels in postmenopausal women. Fertil Steril 2003; 79:455-6.

Recebido em: 16/2/2004 Aceito com modificações em: 19/7/2004 